\title{
Phylogeography of the common toad (Bufo bufo, Lissamphibia: Anura) in Switzerland
}

\author{
Lea E. Waser ${ }^{1}$, Manuel Schweizer ${ }^{1}$, Benedikt R. Schmidt ${ }^{2,3}$, Stefan T. Hertwig ${ }^{1}$
}

${ }^{1}$ Naturhistorisches Museum der Burgergemeinde Bern, Bernastrasse 15, CH-3005 Bern, Switzerland

${ }^{2}$ Koordinationsstelle für Amphibien- und Reptilienschutz in der Schweiz (karch), Passage Maximilien-de-Meuron 6, CH-2000 Neuchâtel, Switzerland

${ }^{3}$ Institut für Evolutionsbiologie und Umweltwissenschaften, Universität Zürich, Winterthurerstrasse 190, CH-8057 Zürich, Switzerland

\section{Supplementary Material}




\section{Material and methods}

\section{Laboratory protocols}

A total of $25 \mu 1$ PCR reaction volume was used, containing 12.5 $\mu$ l GoTaq ${ }^{\circledR}$ Green Master Mix (Promega, Madison, Wisconsin, USA), 1-2 $\mu$ l each of

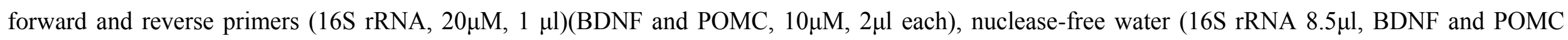
$6.5 \mu \mathrm{l}$ ) and $2 \mu \mathrm{l}$ template DNA. PCR conditions for the $16 \mathrm{~S} \mathrm{rRNA}$ analysis were as follows: denaturation at $94^{\circ} \mathrm{C}$ for $2 \mathrm{~min} ; 35 \mathrm{cycles}$ at $94^{\circ} \mathrm{C}$ for $0: 30 \mathrm{~min}$, $48.2^{\circ} \mathrm{C}$ for $0: 30 \mathrm{~min}$, and $72^{\circ} \mathrm{C}$ for $1: 00 \mathrm{~min}$ followed by a final extension at $72^{\circ} \mathrm{C}$ for 5:00 min. Amplification of BDNF was carried out under the following cycling conditions: denaturation at $94^{\circ} \mathrm{C}$ for $2 \mathrm{~min}, 35$ cycles at $94^{\circ} \mathrm{C}$ for $0: 30 \mathrm{~min}, 51.5^{\circ} \mathrm{C}$ for $0: 30 \mathrm{~min}$, and $72^{\circ} \mathrm{C}$ for $1: 00 \mathrm{~min}$, followed by one final extension at $72^{\circ} \mathrm{C}$ for 5:00 min. The conditions for POMC amplification were denaturation at $94^{\circ} \mathrm{C}$ for 2 min, 35 cycles at $94^{\circ} \mathrm{C}$ for $0: 30 \mathrm{~min}$, $56^{\circ} \mathrm{C}$ for $0: 30 \mathrm{~min}$, and $72^{\circ} \mathrm{C}$ for 1:00 min with a final extension at $72^{\circ} \mathrm{C}$ for 5:00 min. PCR products were either excised from gels and cleaned using Wizard ${ }^{\circledR}$ SV Gel and PCR Clean-Up System (Promega) or directly purified using the kit mentioned above.

Bayesian inference (BI) was carried out in MrBayes 3.2 (Huelsenbeck and Ronquist, 2001) with two independent runs of Metropolis-coupled Markov chain Monte Carlo analyses (three heated and one cold chain, a default temperature of 0.2, 20 million generations, a sampling frequency of 2000). The convergence of parameters and the length of the "burn-in" period were checked with Tracer v.1.5 (Drummond and Rambaut, 2008). The first 10\% of samples from the chains were discarded as burn-in. Maximum likelihood (ML) was carried out in RaxML 7.7.1 (Stamatakis, 2006) using the GTR model of sequence evolution with a Gamma model of rate heterogeneity and 100 bootstrap inferences based on the partition scheme evaluated by PartitionFinder. Median-joining networks were constructed using Network 4.6.1.1 (Bandelt et al., 1999). 


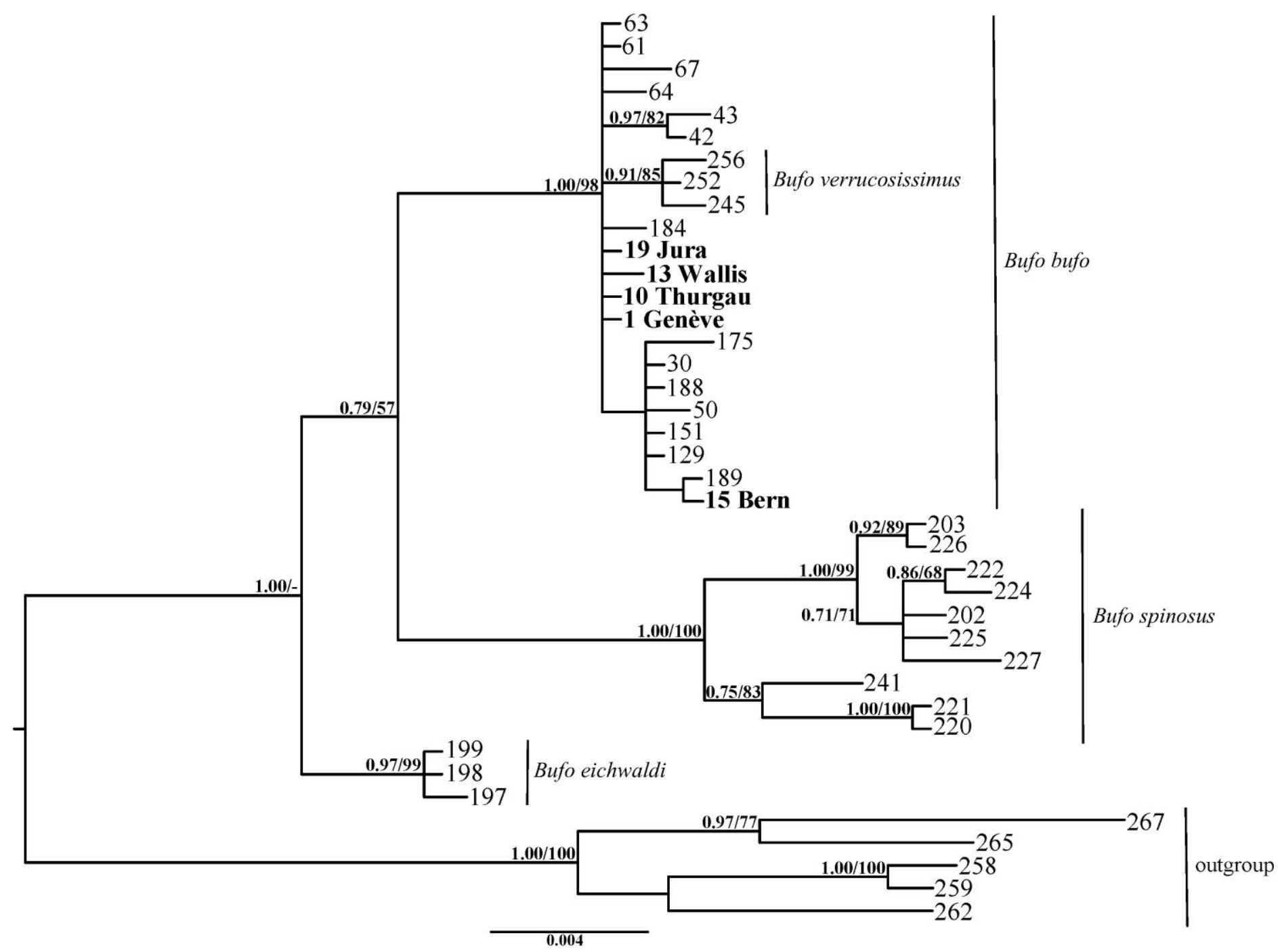

Figure S1. Majority rule consensus tree of the Bayesian inference analysis of the concatenated data set using MrBayes. Support values are Bayesian posterior probabilities and bootstrap values obtained from the maximum likelihood analysis with RAxML. The numbers correspond to the specimens listed in Appendix; Swiss haplotypes (in bold letters) are represented by one specimen only (fig. 1, fig. S4, Appendix). 


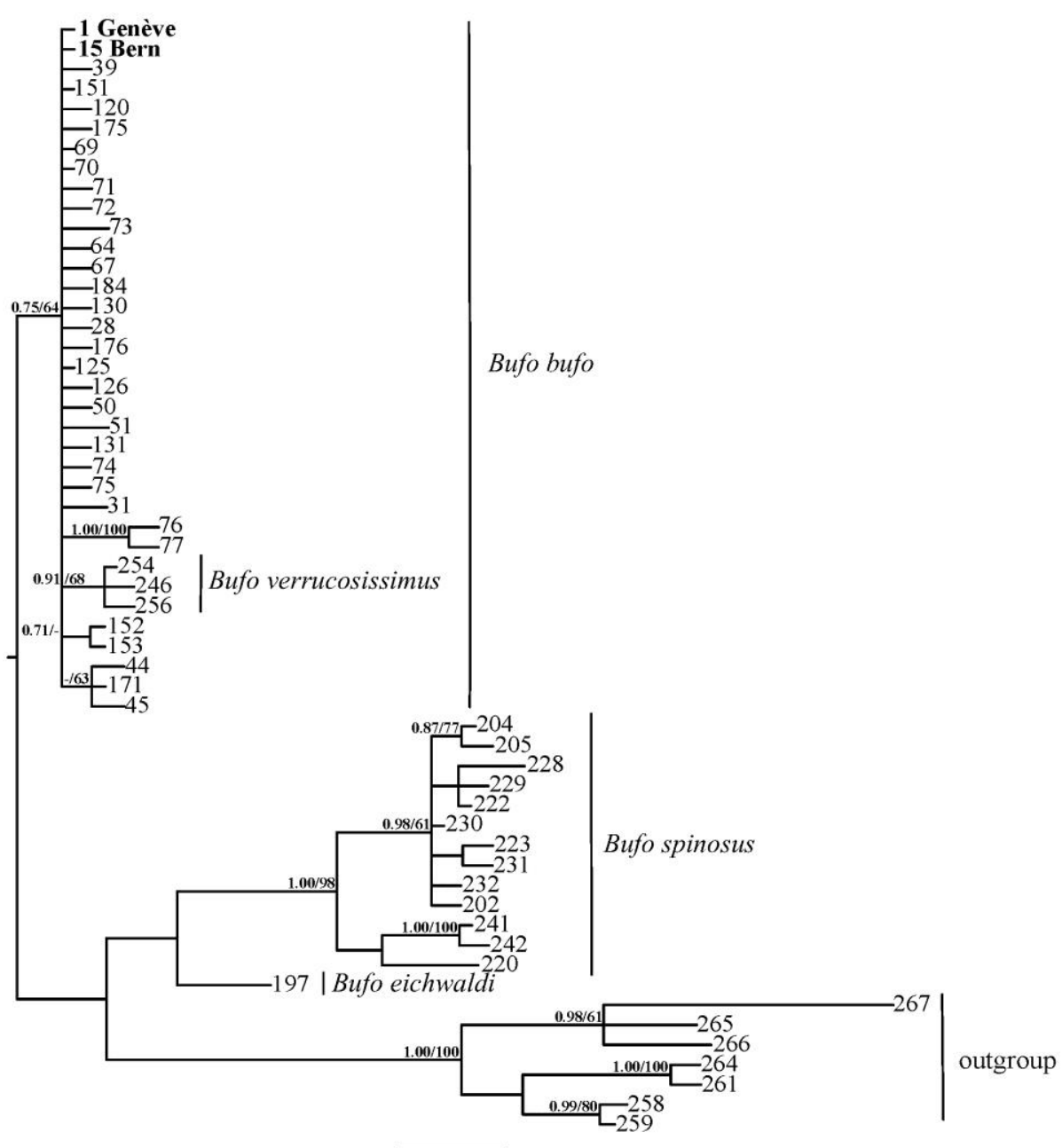

Figure S2. Majority rule consensus tree of the Bayesian inference analysis of the mitochondrial sequences using MrBayes. Support values are Bayesian posterior probabilities and bootstrap values obtained from the maximum likelihood analysis with RAxML. The numbers correspond to the specimens listed in Appendix; Swiss haplotypes (in bold letters) are represented by one specimen only (fig. 1, Appendix). 


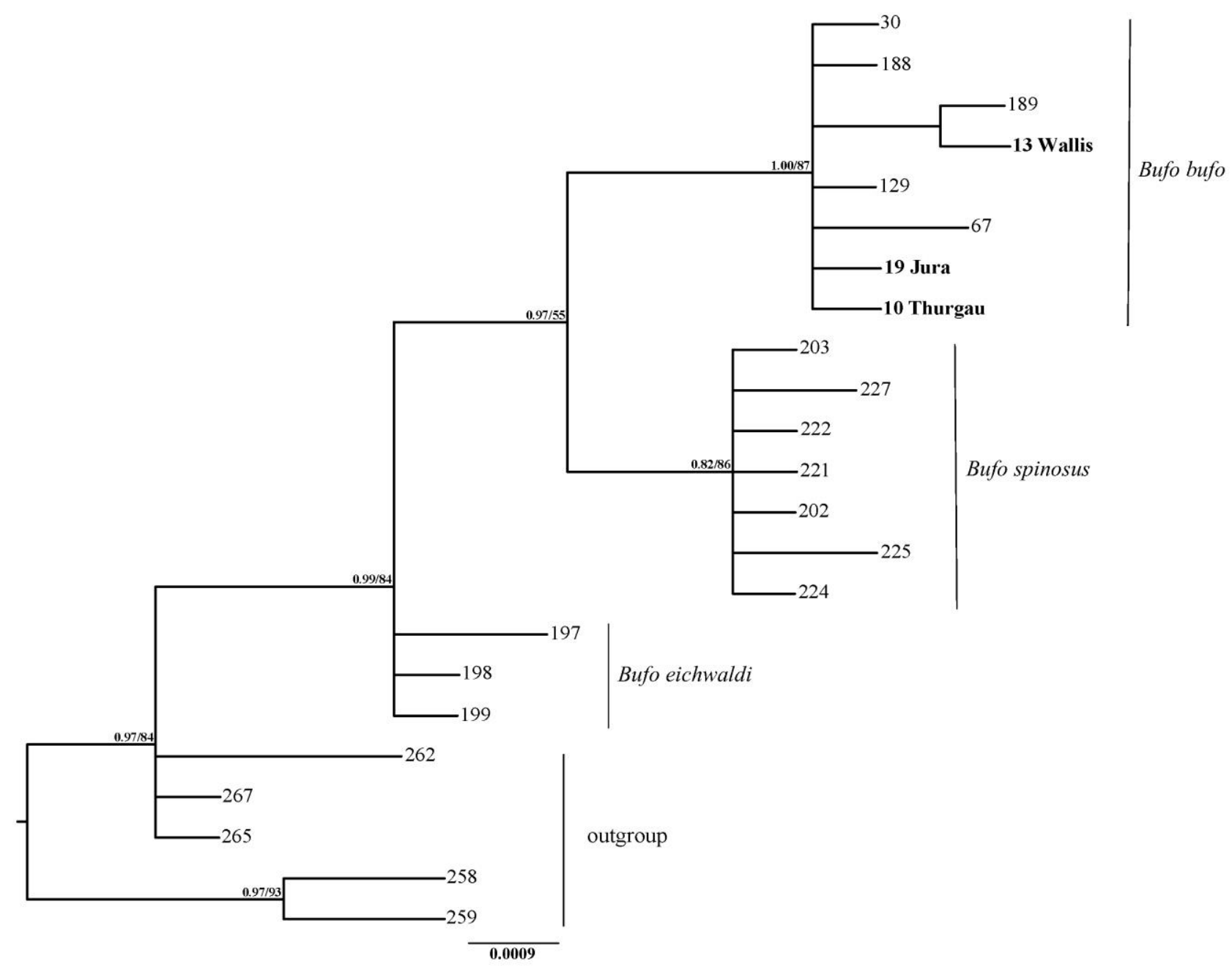

Figure S3. Majority rule consensus tree of the Bayesian inference analysis of the nuclear sequences (BDNF + POMC) using MrBayes. Support values are Bayesian posterior probabilities and bootstrap values obtained from the maximum likelihood analysis with RAxML. The numbers correspond to the specimens listed in Appendix; Swiss haplotypes (in bold letters) are represented by one specimen only (fig. S4, Appendix). 
Network BDNF
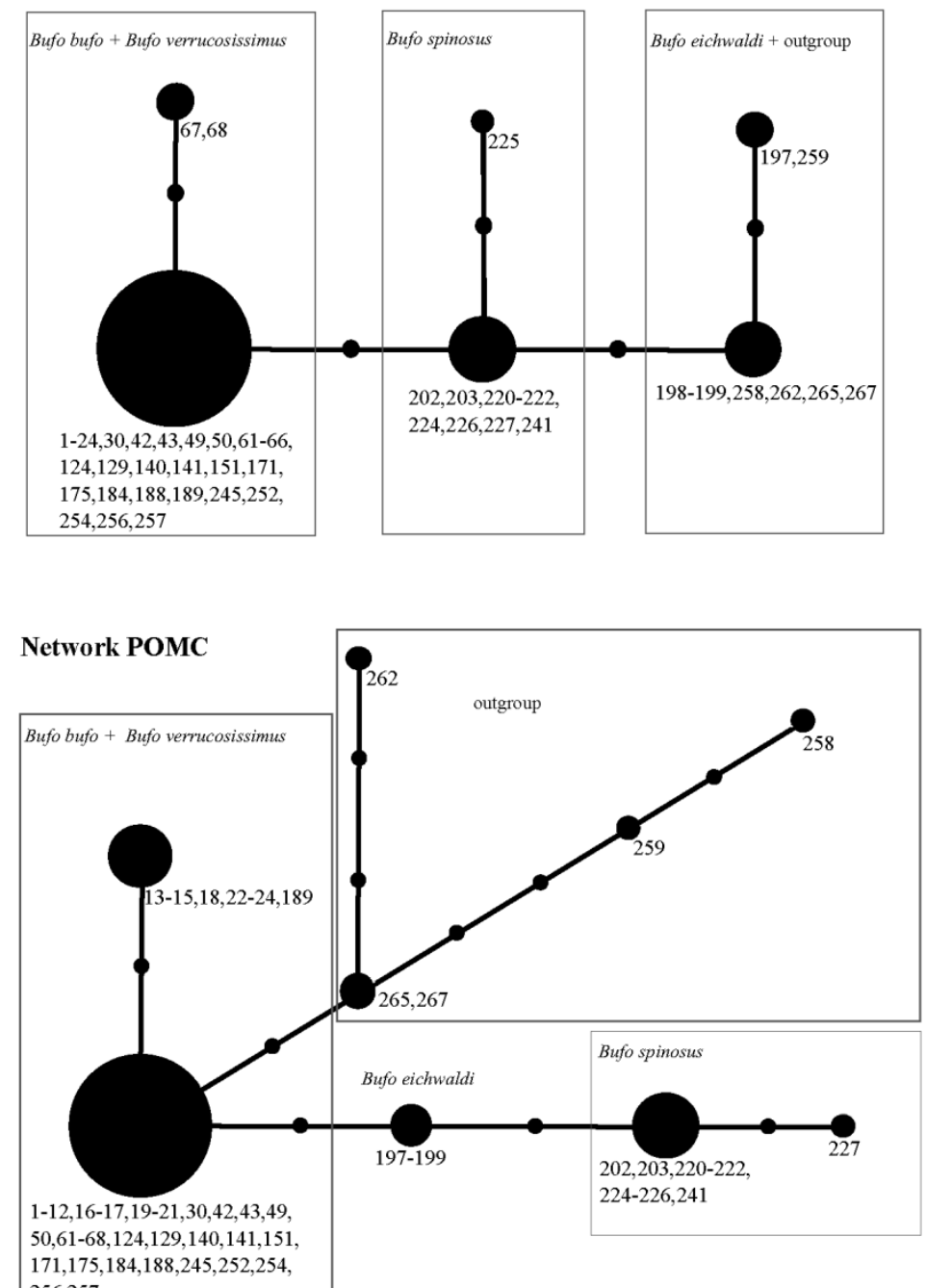

Figure S4. Networks of the nuclear genes BDNF and POMC. Each haplotype is represented by a circle, with width proportional to frequency. Black dots represent single substitution steps. The numbers correspond to the specimens listed in Appendix, specimens 1-24 are from Switzerland. 